$>$ La mise en évidence de séquences immunostimulantes au sein des brins d'ADN en dehors de toute traduction est une découverte récente de la biologie de I'ADN. La présence de motifs CpG, reconnus par un récepteur spécifique (Toll-like receptor 9), induit l'activation des macrophages, des cellules natural killer (NK) et des lymphocytes $B$, et oriente la réponse lymphocytaire $T$ vers le profil Thl. Les propriétés immunostimulantes des motifs CpG ont été utilisées avec succès dans de nombreux modèles animaux de vaccins, d'allergies ou de maladies infectieuses, et plusieurs essais cliniques sont en cours. Dans le cas des cancers, lorsqu'un antigène tumoral est connu, les motifs CpG peuvent être utilisés comme adjuvants dans un cadre vaccinal, ou combinés avec des anticorps monoclonaux. Dans les autres cas, des oligonucléotides de synthèse portant des motifs CpG (CpG-ODN) peuvent être utilisés de façon locale pour stimuler l'immunité innée et favoriser l'émergence d'une réponse immune spécifique. Cet article fait le point sur les progrès récents dans I'utilisation des CpG-ODN en cancérologie. <

\section{Immunothérapie des cancers par oligonucléotides immunostimulants}

\author{
Antoine F. Carpentier
}

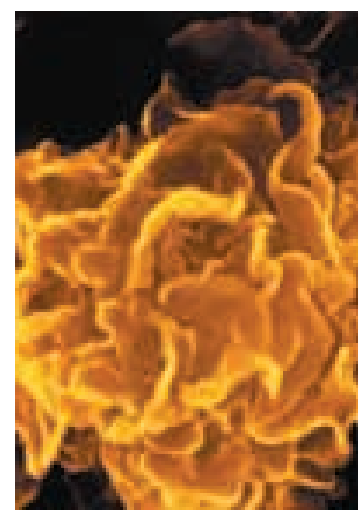

Fédération de Neurologie Mazarin et Inserm U.495, Hôpital de la Salpêtrière, 47, boulevard de l'Hôpital, 75651 Paris Cedex 13, France. antoine.carpentier@

peutiques des CpGpsl.ap-hop-paris.fr ODN en cancérologie, qui font l'objet de cet article, ont été mises en évidence plus tardivement, à la fin des années 1990.

\section{Un acteur important: le TLR9}

La stimulation du système immunitaire requiert la pénétration intracellulaire des CpG-ODN ou de l'ADN bactérien. Cette pénétration ne semble pas dépendre d'un récepteur spécifique, mais se fait par endocytose. Les fragments d'ADN ou les ODN sont ensuite dégradés dans le milieu acide des endosomes, un processus inhibé par la chloroquine ou la bafilomycine A [4]. Les motifs CpG sont reconnus de façon spécifique par un membre de la famille des Toll-like receptors, le TLR9, exprimé au sein des endosomes. L'activité biologique des CpG-ODN est ainsi supprimée chez les souris dont le gène codant pour le TLR9 a été invalidé, et peut être induite dans des cellules TLR9-négatives après leur transfection par $\operatorname{TLR} 9[5,6]$. L'activation intracellulaire après liaison au TLR9 entraîne l'activation des voies des MAP-kinases (mitogen activated protein kinases) et du NFKB (nuclear factor KB) [7].

Chez l'homme, l'expression de TLR9 est restreinte aux lymphocytes $B$ et aux cellules dendritiques plasmocytoïdes ( $p D C)$, alors que son expression chez la souris concerne aussi la lignée myéloïde (macrophages, 
monocytes, cellules dendritiques myéloïdes) [8]. Une expression de TLR9 dans le système nerveux central a également été mise en évidence chez le rat, dans les cellules microgliales [9].

Chez l'homme, comme chez la souris, l'ensemble des lymphocytes B est activé par les CpG-ODN, aboutissant à la sécrétion de cytokines comme l'interleukine-6 (IL-6) ou I'IL-10, à la prolifération cellulaire, à l'inhibition de l'apoptose induite par divers agents et à la sécrétion d'immunoglobulines [2, 10, 11] (Figure 1).

L'activation des pDC humaines, quant à elle, entraîne leur maturation, la sécrétion de nombreuses cytokines comme le TNF $\alpha$ (tumor necrosis factor $\alpha$ ), les interférons $\alpha$ ou $\gamma$ (IFN $\alpha$ ou $\gamma$ ), I'IL-6 ou I'IL-12, et l'expression de molécules de costimulation (CD40, CD80, CD86) et de CCR7, un récepteur contrôlant la migration vers les zones T ganglionnaires [7, 12]. Alors que les pDC sont directement activées par les CpG-ODN, l'activation des cellules dendritiques myéloïdes humaines se fait indirectement, par le biais de cytokines [13]. L'activation des lymphocytes T par les CpG-ODN dépend des cytokines sécrétées par les cellules dendritiques. La sécrétion d'IL-12 et d'IFN $\gamma$ oriente la réponse vers le profil Thl, et peut même transformer une réponse Th2 en Th1 [14]. De plus, les cellules dendritiques peuvent alors activer les lymphocytes CD8, indépendamment des lymphocytes CD4 helper [15].

Les cellules NK sont activées par les CpG-ODN de façon indirecte, par le biais de cytokines comme le TNF $\alpha$ et I'IL-12, et non directement, contrairement à ce qui fut initialement publié.

\section{Les différentes classes de CPG-ODN}

L'activité biologique des motifs $\mathrm{CpG}$ dépend des bases adjacentes en 5' et 3 ', et de la nature chimique du squelette de l'oligonucléotide. Trois familles de CpGODN sont ainsi caractérisées (Tableau I) [7, 16].

Le type B (ou K), le plus classique, se caractérise par une forte activation des lymphocytes $B$ et des cellules dendritiques, mais par une faible sécrétion d'IFN $\alpha$ par les pDC. Certaines séquences, qualifiées d'optimales, présentent une spécificité d'espèce (5'-GTCGTT chez I'homme, 5'-GACGTT chez la souris), mais il existe des séquences (5'-AACGTT ou 5'-GACGTC) possédant une excellente activité immunostimulante dans les deux espèces. La répétition d'hexamères $\mathrm{CpG}$ au sein d'un même ODN augmente franchement son activité $[7,17]$. Les ODN de type $A$ (ou $D$ ) se caractérisent par une faible activation des lymphocytes $B$, mais par une forte activation des cellules NK et par une sécrétion d'IFN $\alpha$ par les PDC. Les ODN de type $C$, plus récemment décrits, combinent les propriétés des deux précédents types.

L'activité biologique de l'ensemble des CpG-ODN, quel que soit leur type, dépend de l'expression de TLR9; la raison de ces différences d'activité au moyen d'un même récepteur n'est pas élucidée.

\section{Applications des CPG-ODN en cancérologie}

Le fort pouvoir immunostimulant des CpG-ODN ouvre plusieurs stratégies thérapeutiques dans les cancers. L'utili-

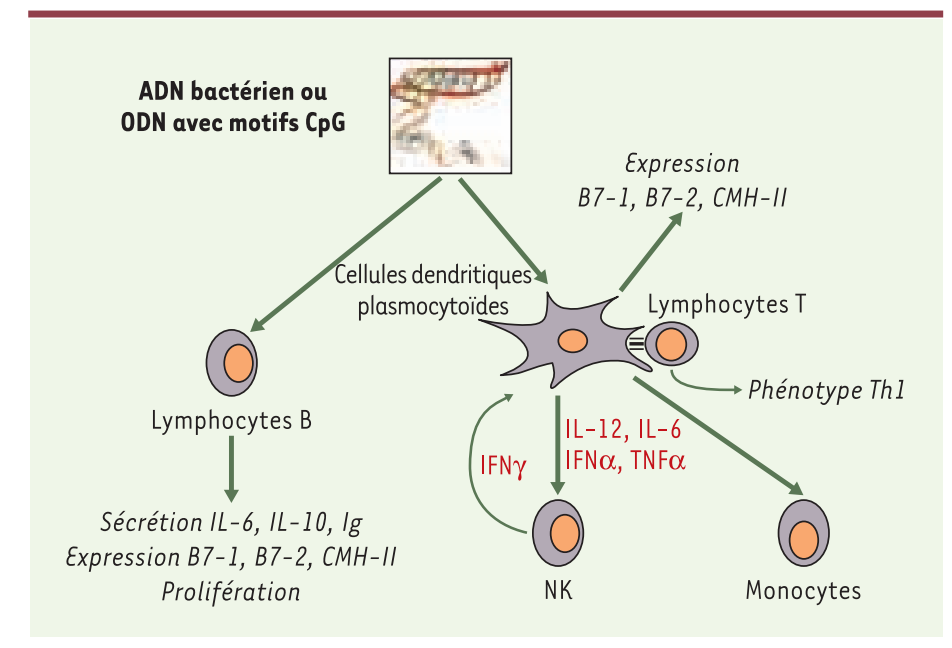

Figure 1. L'ADN bactérien et les CPG-ODN ont une action directe sur les lymphocytes $B$ et les cellules dendritiques plasmocytoïdes chez les mammifères. Les CpG-ODN ont, sur les lymphocytes $B$, un effet mitogène et inducteur de cytokines comme l'interleukine-6 (IL-6) ou l'IL-10. Après activation par les CpG$O D N$, les cellules dendritiques sécrètent de nombreuses cytokines comme le TNF $\alpha$, I'IL-6 ou I'IL-12, favorisant ainsi une réponse des lymphocytes T de type Th1. Les cellules NK (natural killer) sont activées de façon indirecte, mais augmentent la réponse immunitaire par la sécrétion d'interféron $\gamma$ (IFN $\gamma$ ).

\begin{tabular}{lccc}
\hline Type & Effets immunostimulants & Exemples & Références \\
A (D) & Faible activation LB & GG TGCATCGATGCAG GGGGG & [7] \\
& Forte activation NK et pDC & (ODN mixte phosphodiester [en italique ] & \\
& Forte sécrétion d'IFN $\alpha$ par les pDC & et phosphorothioate) & [16] \\
B (K) & Faible activation NK & ODN 2006: & [22] \\
& Forte activation LB et pDC & TCGTCGTTTGTCGTTTGTCGTT & \\
& Faible sécrétion d'IFN $\alpha$ par les pDC & CpG-28: TAAACGTTATAACGTATGACGTCAT & (ODN phosphorothioate) \\
C & Forte activation LB, NK et pDC & TCGTCGTTCGACGACGTTGAT & \\
& Forte sécrétion d'IFN $\alpha$ par les pDC & (ODN phosphorothioate) &
\end{tabular}

Tableau I. Familles de CPG-ODN décrites dans la littérature. LB: lymphocytes B; NK: cellules natural killer; pDC: cellules dendritiques plasmocytoïdes; IFN $\alpha$ : interféron $\alpha$. 
sation des CpG-ODN permet en effet d'activer les deux composantes de la réponse immune: son amorçage et son efficacité. La stimulation directe des cellules dendritiques par les CpGODN favorise la sélection par le système immunitaire d'un antigène adéquat avec une réponse immune de type Thl. Ensuite, l'activation des macrophages et des cellules NK permet d'accroître la cytotoxicité antitumorale, soit directe, soit par le biais des anticorps (antibody-dependant cell cytotoxicity, $A D C C$ ). De plus, grâce à la sécrétion locale d'IFN $\gamma$, l'expression généralement faible du CMH-I (complexe majeur d'histocompatibilité) par les cellules tumorales peut être induite, les rendant plus sensibles à la cytotoxicité des lymphocytes $\mathrm{T}$.

Schématiquement, on peut distinguer deux approches dans le traitement des cancers par les CpG-ODN : I'utilisation des CpGODN seuls, par voie locale, pour favoriser l'émergence d'une réponse immunitaire (immunisation in situ), ou l'utilisation combinée des CpG-ODN avec un antigène ou un anticorps monoclonal, lorsque ceux-ci sont connus, ou avec des cellules dendritiques (immunisation à distance). La quasi-totalité des travaux dont les résultats sont rapportés ci-dessous ont utilisé les CpG-ODN de type $B$, les plus anciennement connus.

\section{Utilisation des CPG-ODN seuls}

L'efficacité d'une telle approche avait été suggérée par l'utilisation d'extraits nucléaires de Mycobacterium bovis (MY-1), dont l'injection permettait la régression de tumeurs établies chez la souris [1]. L'activité de MY-1 étant dépendante de la présence d'ADN bactérien, il est probable que l'activité biologique observée dépendait des motifs $C p G$ contenus dans cet $A D N$ bactérien. Ce concept a finalement été démontré par notre équipe dans un modèle animal de neuroblastome sous-cutané, où l'injection péritumorale d'ODN synthétiques contenant des motifs $C p G$ induisait une régression tumorale chez la moitié des animaux, et amorçait une réponse immune protectrice prolongée [18].

De nombreuses publications sont venues corroborer ces résultats, dans presque tous les modèles de tumeurs solides [9]. Par exemple, dans un modèle de gliome intracérébral (CNS1), plus de $85 \%$ des rats furent guéris par une seule injection de CpG-ODN [19].

La situation est probablement plus complexe pour les hémopathies de type B, puisque les CpG-ODN ont un effet direct sur ces cellules. Les CpG-ODN peuvent augmenter l'immonogénicité de ces cellules par induction de molécules de surface comme le CD80, le CD86 ou les CMH de classe I et II. D'un autre coté, les CpG-ODN stimulent la prolifération des cellules lymphomateuses, et les protègent de l'apoptose induite par de nombreux agents. Globalement, un effet positif a été rapporté dans quelques modèles animaux, effet très modeste dans un cas [20, 21].

Aucune toxicité directe des CpG-ODN sur les cellules tumorales n'a été mise en évidence in vitro. En revanche, la plupart des études ont souligné le rôle conjoint des systèmes immunitaires inné non spécifique et spécifique dans le rejet tumoral. La déplétion in vivo des cellules NK diminue ou abolit les effets antitumoraux des CpG-ODN [18]. Les cellules NK n'étant pas une cible première des $C p G-0 D N$, les macrophages infiltrants et les cellules dendritiques interviennent probablement en amont de cette activation [21, 22]. L'implication de l'immunité spécifique est clairement démontrée par l'efficacité réduite des CpG-ODN dans les souris nude ou SCID [19]. Les lymphocytes CD8 sont nécessaires dans la plupart des modèles animaux, alors que la déplétion en cellules CD4 semble avoir moins d'impact $[22,23]$, probablement parce que les CpG-ODN permettent l'activation directe des lymphocytes CD8 par les cellules dendritiques, de façon indépendante des lymphocytes CD4 [15]. Cependant, l'existence d'une mémoire immunitaire à long terme, rapportée dans l'ensemble des modèles, suggère un rôle joué par les lymphocytes CD4 mémoire. De façon intéressante, il a été rapporté que les $\mathrm{pDC}$ infiltrantes des tumeurs $\mathrm{ORL}$ chez I'homme étaient moins sensibles aux CpG-ODN de type A que les pDC sanguines du même patient. Cette résistance s'expliquerait en partie par la sécrétion locale d'IL-10 par les cellules tumorales [24]. Dans un modèle animal, la combinaison de CpG et d'anticorps anti-IL-10 a d'ailleurs révélé l'existence d'un effet synergique [25].

En conclusion, il faut souligner que l'activation de l'immunité non spécifique est locale et persiste tant que le traitement est administré, à l'inverse de l'immunité spécifique. Ainsi, l'immunité non spécifique joue probablement un rôle important dans la phase initiale du traitement, avant qu'une immunité spécifique soit amorcée. Les résultats préliminaires d'un essai clinique mené sur des sujets atteints de mélanome montrent un réponse réelle au site d'injection, mais pas à distance [26]. La capacité des CpG-ODN à induire une bonne réponse spécifique sera donc déterminante pour leur avenir clinique, en particulier pour les cancers métastatiques.

\section{CPG-ODN en association avec des antigènes,} des anticorps ou des cellules dendritiques

Les CpG-ODN sont des adjuvants très efficaces avec la plupart des antigènes, sauf si ceux-ci sont de nature purement polysaccharidique [27]. Les CpG-ODN sont ainsi les adjuvants les plus efficaces pour l'induction des réponses immunitaires Th1 cytotoxiques avec lymphocytes T CD4 et CD8 spécifiques de l'antigène [28, 29]. Dans un modèle de mélanome exprimant I'épitope OVA, par exemple, les CpG-ODN ont augmenté la réponse CTL (réponse des lymphocytes $T$ cytolytiques) après immunisation avec des peptides synthétiques et ont permis la régression tumorale [30]. La conjugaison directe entre l'antigène et les $C p G-0 D N$ permet de réduire la quantité d'antigène nécessaire [31]. L'efficacité des CpG-ODN est encore améliorée par leur combinaison avec d'autres adjuvants comme l'hydroxyde d'aluminium, le QS21 (saponine), le MPL (lipide A monophosphorylé) ou le GM-CSF (granulocye-macrophage colony-stimulating factor) $[29,32,33]$. 
La combinaison de CpG-ODN et d'anticorps monoclonaux pourrait se révéler intéressante. Les $C p G-0 D N$ augmentent la cytotoxicité relayée par les anticorps ( $A D C C)$ grâce à la stimulation des macrophages et des cellules NK. Dans un modèle animal de lymphome, une telle combinaison permet de prévenir la croissance tumorale [34]. Plusieurs essais cliniques utilisant cette association ont débuté récemment, avec de l'Herceptin ${ }^{\circledR}$ dans les cancers du sein ou du Rituxan ${ }^{\circledR}$ dans les lymphomes non-hodgkiniens.

Plus récemment, une approche originale a utilisé les CpG-ODN comme inducteur au niveau de la cellule tumorale de l'expression d'une molécule contre laquelle un anticorps monoclonal existe. Les CpG-0DN induisent l'expression du CD25 (IL-2R) à la surface d'une lignée lymphomateuse $B$, rendant ainsi ces cellules plus sensibles à l'action d'une immunotoxine anti-CD25 [35]. Malheureusement, les cibles antigéniques sont rarement déterminées dans les cancers, limitant l'intérêt pratique de telles stratégies thérapeutiques. De plus, la sélection spontanée d'un clone n'exprimant pas l'antigène permettrait à la tumeur d'échapper à un tel traitement.

Les CpG-ODN permettent la maturation et l'activation des cellules dendritiques, et favorisent l'activation de lymphocytes CD8 cytotoxiques in vivo. La stimulation par des CpG-ODN de cellules dendritiques en présence de lymphocytes $T$ helpers et de cellules de lymphome A20 a ainsi permis l'expansion d'un grand nombre de lymphocytes Thl spécifiques de la tumeur, capables d'éradiquer chez l'animal le lymphome établi [36]. Dans un modèle de carcinome colique, la combinaison de CpG par voie locale et d'immunisation à distance par des cellules dendritiques chargées avec des antigènes tumoraux induit la régression de grandes tumeurs établies, alors que chacune des approches prises séparément n'a qu'une efficacité limitée [37]. L'utilisation des CpG-ODN avec les cellules dendritiques, qui s'affranchit du problème de la sélection des antigènes tumoraux, devrait bientôt rentrer en phase clinique.

\section{Conclusions et perspectives}

La mise en évidence des propriétés immunologiques des motifs $C p G$ au sein des ODN résulte d'une découverte récente et tout à fait inattendue de la biologie de l'ADN.

Plusieurs essais cliniques sont actuellement en cours avec des CpG-ODN (de type B), seuls ou en association avec des anticorps monoclonaux, dans les mélanomes, les lymphomes, les cancers du sein, ou les cancers du rein. Un essai clinique dans les glioblastomes en récidive a débuté à l'hôpital de la Salpêtrière (Paris, France) en février 2003.

Dans les prochaines années, l'accent sera probablement mis sur la définition de séquences immunostimulantes optimales et de formes galéniques adaptées. Par exemple, une forme à libération prolongée locale sera particulièrement justifiée lorsqu'une activation prolongée de l'immunité non spécifique est désirée (cancers locaux, sensible aux cellules NK). À l'inverse, en cas de cancers métastatiques, la capacité du traitement à induire une immunité spécifique sera cruciale.

Enfin, il est évident que les combinaisons de CpG-ODN avec la radiothérapie ou la chimiothérapie pourront se révéler intéressantes $[11,38]$. Une réduction de la masse tumorale permettrait le relargage d'antigènes tumoraux et favoriserait l'éradication par le système immunitaire de la masse tumorale résiduelle. $\diamond$

\section{REMERCIEMENTS}

Ce travail à été soutenu par l'Inserm, l'Assistance Publique-Hôpitaux de Paris et l'Université Paris V.

\section{SUMMARY}

\section{Cancer immunotherapy with CPG-ODN}

Bacterial DNA and synthetic oligodeoxynucléotides containing CpG motifs (CpG-ODN) are the ligands for the Toll-like receptor 9 (TLR9), which is expressed by B-lymphocytes and a subset of dendritic cells. CpG-ODN are strong activators of both innate and specific immunity, and drive the immune response towards the Thl phenotype. Given the promising results obtained in several experimental models of allergies or infections, CpG-ODN are now entering clinical trials for these diseases. In cancer, promising approaches combined CpG-ODN with tumor antigens, monoclonal antibodies or dendritic cells. When no relevant tumor antigen is known, CpG-ODN can be used alone to activate locally the innate immunity and trigger a tumor-specific immune response, overcoming the need for the identification of a tumoral antigen. Preclinical models have shown impressive results and several clinical trials are on-going worldwide in melanoma, lymphoma, renal carcinoma, breast cancer and glioblastoma. $\diamond$

\section{RÉFÉRENCES}

1. Tokunaga T, Yamamoto H, Shimada S, et al. Antitumor activity of deoxyribonucleic acid fraction from Mycobacterium bovis BCG. I. Isolation, physicochemical characterization, and antitumor activity.

J Natl Cancer Inst 1984; 72: 955-62.

2. Krieg AM, Yi AK, Matson S, et al. CpG motifs in bacterial DNA trigger direct B-cell activation. Nature 1995; 374 : 546-9.

3. Yamamoto S, Yamamoto T, Kataoka T, et al. Unique palindromic sequences in synthetic oligonucleotides are required to induce IFN and augment IFN-mediated natural killer activity. J Immunol 1992; 148: 4072-6.

4. Hacker H. Signal transduction pathways activated by CpG-DNA. Curr Top Microbiol Immunol 2000; 247: 77-92.

5. Hemmi H, Takeuchi 0, Kawai T, et al. Toll-like receptor recognizes bacterial DNA. Nature $2000 ; 408: 740-5$.

6. Takeshita F, Leifer CA, Gursel I, et al. Cutting edge: role of Toll-like receptor 9 in CpG DNA-induced activation of human cells. J Immunol 2001; 167: 3555-8.

7. Klinman DM. Immunotherapeutic uses of $\mathrm{CpG}$ oligodeoxynucleotides. Nat Rev Immunol 2004; 4 : 249-59.

8. Hornung V, Rothenfusser S, Britsch S, et al. Quantitative expression of toll-like receptor 1-10 mRNA in cellular subsets of human peripheral blood mononuclear cells and sensitivity to CpG oligodeoxynucleotides. J Immunol 2002; 168: 4531-7.

9. Carpentier AF, Auf G, Delattre JY. CpG-oligonucleotides for cancer immunotherapy: review of the literature and potential applications in malignant glioma. Front Biosci 2003; 8: ह115-27.

10. Jung J, Yi AK, Zhang $X$, et al. Distinct response of human B cell subpopulations in recognition of an innate immune signal, CpG DNA. J Immunol 2002; 169: 2368-73.

11. Sparwasser T, Koch ES, Vabulas RM, et al. Bacterial DNA and immunostimulatory $\mathrm{CPG}$ oligonucleotides trigger maturation and activation of murine dendritic cells. Eur J Immunol 1998; 28: 2045-54. 
12. Ban $\varepsilon$, Dupre L, Hermann $\varepsilon$, et al. CpG motifs induce Langerhans cell migration in vivo. Int Immunol 2000; 12: 737-45.

13. Krug A, Towarowski A, Britsch S, et al. Toll-like receptor expression reveals CpG DNA as a unique microbial stimulus for plasmacytoid dendritic cells which synergizes with CD40 ligand to induce high amounts of IL-12. Eur J Immunol 2001; 31: 3026-37.

14. Kline JN, Waldschmidt TJ, Businga TR, et al. Modulation of airway inflammation by $\mathrm{CpG}$ oligodeoxynucleotides in a murine model of asthma. J Immunol 1998; 160: 2555-9.

15. Cho HJ, Takabayashi K, Cheng PM, et al. Immunostimulatory DNA-based vaccines induce cytotoxic lymphocyte activity by a T-helper cell-independent mechanism. Nat Biotechnol 2000; 18: 509-14.

16. Krieg AM. Antitumor applications of stimulating toll-like receptor 9 with $C p G$ oligodeoxynucleotides. Curr Oncol Rep 2004; 6: 88-95.

17. Hartmann G, Weeratna RD, Ballas ZK, et al. Delineation of a CpG phosphorothioate oligodeoxynucleotide for activating primate immune responses in vitro and in vivo. J Immunol 2000; 164: 1617-24

18. Carpentier AF, Chen L, Maltonti F, Delattre Jy. Oligodeoxynucleotides containing CpG motifs can induce rejection of a neuroblastoma in mice. Cancer Res 1999; 59: 5429-32.

19. Carpentier AF, Xie J, Mokhtari K, Delattre JY. Successful treatment of intracranial gliomas in rat by oligodeoxynucleotides containing CpG motifs. Clin Cancer Res $2000 ; 6: 2469-73$

20. Decker T, Schneller F, Sparwasser T, et al. Immunostimulatory CpG-oligonucleotides cause proliferation, cytokine production, and an immunogenic phenotype in chronic lymphocytic leukemia B cells. Blood 2000; 95: 999-1006.

21. Hafner M, Zawatzky R, Hirtreiter C, et al. Antimetastatic effect of CpG DNA mediated by type I IFN. Cancer Res 2001; 61: 5523-8.

22. Lanuti M, Rudginsky S, Force SD, et al. Cationic lipid/bacterial DNA complexes elicit adaptive cellular immunity in murine intraperitoneal tumor models. Cancer Res 2000; 60: 2955-63.

23. Kawarada Y, Ganss R, Garbi N, et al. NK- and $C D 8^{+} T$ cell-mediated eradication of established tumors by peritumoral injection of $\mathrm{CpG}$-containing oligodeoxynucleotides. J Immunol 2001; 167: 5247-53

24. Hartmann $\varepsilon$, Wollenberg $B$, Rothenfusser $S$, et al. Identification and functional analysis of tumor-infiltrating plasmacytoid dendritic cells in head and neck cancer. Cancer Res 2003; 63: 6478-87.

25. Vicari AP, Chiodoni C, Vaure C, et al. Reversal of tumor-induced dendritic cell paralysis by $\mathrm{CpG}$ immunostimulatory oligonucleotide and anti-interleukin 10 receptor antibody. J Exp Med 2002; 196: 541-9.

26. Trefzer U, Kors C, Pelzer K, et al. Preliminary results of a phase I trial of intralesional injection of $\mathrm{CpG}$-DNA in basal cell carcinoma and melanoma. $2^{\text {nd }}$ International Symposium Activating immunity with CPG Oligos. Amelia Island, Florida, 7-10 octobre 2001

27. Krieg AM. CpG motifs in bacterial DNA and their immune effects. Annu Rev Immunol $2002 ; 20: 709-60$

28. Zwaveling S, Ferreira Mota SC, Nouta J, et al. Established human papillomavirus type 16-expressing tumors are effectively eradicated following vaccination with long peptides. J Immunol 2002; 169: 350-8.

29. Miconnet I, Koenig S, Speiser D, et al. CpG are efficient adjuvants for specific CTL induction against tumor antigen-derived peptide. I Immunol 2002; 68: 1212-8.

30. Davila $\varepsilon$, Celis $\varepsilon$. Repeated administration of cytosine-phosphorothiolated guanine containing oligonucleotides together with peptide/protein immunization results in enhanced CTL responses with anti-tumor activity. J Immunol 2000; 165: 539-47.

31. Tighe H, Takabayashi K, Schwartz D, et al. Conjugation of immunostimulatory DNA to the short ragweed allergen amb a $l$ enhances its immunogenicity and reduces its allergenicity. J Allergy Clin Immunol 2000; 106: 124-34.

32. Kim SK, Ragupathi G, Cappello S, et al. Effect of immunological adjuvant combinations on the antibody and T-cell response to vaccination with MUC1-KLH and GD3-KLH conjugates. Vaccine $2000 ; 19: 530-7$.

33. Liu HM, Newbrough SE, Bhatia SK, et al. Immunostimulatory CpG oligodeoxynucleotides enhance the immune response to vaccine strategies involving granulocyte-macrophage colony-stimulating factor. Blood 1998; 92 : $3730-6$

34. Warren TL, Dahle CE, GJ Weiner. CpG oligodeoxynucleotides enhance monoclonal antibody therapy of a murine lymphoma. Clin Lymphoma 2000; 1: 57-61.

35. Decker T, Hipp S, Kreitman RJ, et al. Sensitization of B-cell chronic lymphocytic leukemia cells to recombinant immunotoxin by immunostimulatory phosphorothioate oligodeoxynucleotides. Blood 2002; 99: 1320-6.

36. Egeter $O R$, Mocikat $K$, Ghoreschi $A$, et al. Eradication of disseminated lymphomas with CpG-DNA activated Thelper type 1 cells from nontransgenic mice. Cancer Res $2000 ; 60: 1515-20$

37. Heckelsmiller K, Beck S, Rall K, et al. Combined dendritic cell and $\mathrm{CpG}$ oligonucleotide-based immune therapy cures large murine tumors that resist chemotherapy. Eur J Immunol 2002; 32: 3235-45.

38. Weigel BJ, Rodeberg DA, Krieg AM, Blazar BR. CpG oligodeoxynucleotides potentiate the antitumor effects of chemotherapy or tumor resection in an orthotopic murine model of rhabdomyosarcoma. Clin Cancer Res 2003; $9:$ 3105-14.

\section{ATLAS DE POCHE : UNE COLLECTION D'OUVRAGES PRATIQUES ET INDISPENSABLES AU FORMAT DE POCHE QUI INTEGRENT LES DONNEES LES PLUS RÉCENTES DE LA RECHERCHE.}

\section{NËUROLOEI \\ ATLAS DE POCHE DE NEUROLOGIE}

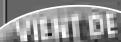

pMEAIRE

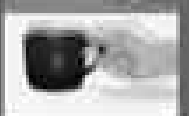

2004 - un volume broché

448 pages, 200 illustrations.

Reinhardt ROHKAMM

En 450 pages et 200 illustrations : tout ce qu'il convient de savoir en neurologie $\bullet$ les bases anatomiques et physiologiques du $\mathrm{SN}$ et des muscles $\bullet$ les signes fonctionnels, les symptômes et les signes cliniques des maladies du SN, les indices d'une atteinte spécifique du SNC, SNP ou des muscles $\bullet$ les tableaux cliniques des maladies neurologiques: causes, symptômes, données de l'examen clinique et des investigations complémentaires, diagnostics différentiels et indications thérapeutiques $\bullet$ les tableaux placés en annexe donnent une vue d'ensemble des éléments indispensables à la pratique clinique quotidienne.

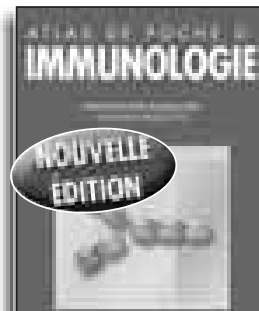

ATLAS DE POCHE D'IMMUNOLOGIE

G. BURMESTER et A. PEZZUTTO

La réussite d'un pari pédagogique : toutes les connaissances nécessaires en immunologie en 325 pages et 400 illustrations dont 135 planches couleur $\bullet$ les notions de base : lymphocytes $B$ et $T$, interactions cellulaires, système HLA, défenses non spécifiques, tolérance, auto-immunité $\bullet$ les modes d'exploration de l'immunité : réactions antigène-anticorps, immunités cellulaire et humorale, apport de la biologie moléculaire

2004 - 2e édition, un volume broché,

325 pages,

400 illustrations. - les applications médicales de l'immunologie et les pathologies depuis les déficits immunitaires jusqu'aux affections immunitaires endocriniennes, en passant par l'asthme, les cancers, les lymphomes, les vaccinations.

\section{ET COMME DANS TOUS LES VOLUMES DE CETTE COLLECTION}

la liaison du texte et de l'image en vis à vis, la rédaction claire et pratique, les illustrations en couleurs, ajoutent à la lisibilité de l'ouvrage et font de ces guides pratiques, exhaustifs et actuels des outils indispensables à l'étudiant, au praticien et à tout médecin soucieux d'actualiser ses connaissance dans ces disciplines.

\section{En vente chez votre libraire spécialisé, sur notre site www. medecine.flammarion.com ou par correspondance.}

\section{BON DE COMMANDE à retourner à}

FLAMMARION MÉDECINE - 4, rue Casimir Delavigne - 75006 PARIS

NOM

Prénom :

Adresse :

Code postal :

Ville:

Je commande et je régle ci-joint par chèque bancaire à l'ordre de Flammarion (une facture acquittée sera jointe au colis)

$\square$ Atlas de poche de neurologie au prix unitaire $T C$ de $45.00 €+5.00 €$ de participation aux frais de port, soit.

$\neg$ Atlas de poche d'immunologie $-2^{\mathrm{e}}$ édition au prix unitaire TTC de $45.00 €+5.00 €$ de participation aux frais de port, soit ..........5.00 $€$ 